\title{
2017 EULAR recommendations for a core data set to support observational research and clinical care in rheumatoid arthritis
}

\author{
Helga Radner, ${ }^{1}$ Katerina Chatzidionysiou, ${ }^{2,3}$ Elena Nikiphorou, ${ }^{4}$ Laure Gossec, 5,6 \\ Kimme L Hyrich, ${ }^{7}$ Condruta Zabalan, ${ }^{8}$ Yvonne van Eijk-Hustings, ${ }^{9}$ Paula R Williamson, ${ }^{10}$ \\ Andra Balanescu, ${ }^{11}$ Gerd R Burmester, ${ }^{12}$ Loreto Carmona, ${ }^{13}$ Maxime Dougados, ${ }^{14}$ \\ Axel Finckh, ${ }^{15}$ Glenn Haugeberg ${ }_{1}^{16,17}$ Merete Lund Hetland ${ }^{18,19}{ }^{\text {Susan Oliver, }}{ }^{20}$ \\ Duncan Porter, ${ }^{21}$ Karim Raza, ${ }^{22,23,24}$ Patrick Ryan, ${ }^{25,26}$ Maria Jose Santos, ${ }^{27}$ \\ Annette van der Helm-van Mil, ${ }^{28,29}$ Piet van Riel, ${ }^{30}$ Gabrielle von Krause, $^{31}$ \\ Jakub Zavada, ${ }^{32,33}$ William G Dixon, ${ }^{34}$ Johan Askling ${ }^{2,3}$
}

\section{Handling editor Tore K Kvien}

- Additional material is published online only. To view please visit the journal online (http://dx.doi.org/10.1136/ annrheumdis-2017-212256).

For numbered affiliations see end of article.

\section{Correspondence to}

Dr Helga Radner, Department of Internal Medicine III, Division of Rheumatology, Medical University Vienna, Vienna 1090, Austria;

helga.radner@meduniwien.

ac.at

Received 19 August 2017 Revised 9 December 2017

Accepted 15 December 2017 Published Online First

4 January 2018
Check for updates

To cite: Radner $\mathrm{H}$ Chatzidionysiou K, Nikiphorou $\mathrm{E}$, et al. Ann Rheum Dis

2018;77:476-479.

\section{ABSTRACT}

Personalised medicine, new discoveries and studies on rare exposures or outcomes require large samples that are increasingly difficult for any single investigator to obtain. Collaborative work is limited by heterogeneities, both what is being collected and how it is defined. To develop a core set for data collection in rheumatoid arthritis (RA) research which (1) allows harmonisation of data collection in future observational studies, (2) acts as a common data model against which existing databases can be mapped and (3) serves as a template for standardised data collection in routine clinical practice to support generation of research-quality data. A multistep, international multistakeholder consensus process was carried out involving voting via online surveys and two face-to-face meetings. A core set of 21 items ('what to collect') and their instruments ('how to collect') was agreed: age, gender, disease duration, diagnosis of RA, body mass index, smoking, swollen/tender joints, patient/evaluator global, pain, quality of life, function, composite scores, acute phase reactants, serology, structural damage, treatment and comorbidities. The core set should facilitate collaborative research, allow for comparisons across studies and harmonise future data from clinical practice via electronic medical record systems.

\section{INTRODUCTION}

Research questions of the current era require ever larger study populations which often exceed the number of patients available in individual studies or registries; hence, combined or pooled analyses are often required. Such collaborative work is, however, compromised by heterogeneities in the data collected. ${ }^{1}$ More recently, routinely collected data from electronic medical records (EMR) has the potential to support research, but there is no agreed set of data that would be desirable to collect in order to generate research-quality data if used beyond direct clinical care.

To facilitate collaborative research, standardising items ('what to collect') and their instruments ('how to collect') across studies and data collections is critical. Although guidelines on data reporting and outcome measures have been developed, such core sets have so far pertained to randomised control trials and trial extension studies in rheumatoid arthritis (RA) ${ }^{2}$ and have not been vetted against the feasibility of data collection in routine clinical care or against the needs of observational research. Indeed, any core data set to be implemented in registers, research cohorts or EMR systems need to strike a balance between clinical feasibility and the potential to generate useful, research-quality data.

For these reasons, a European League Against Rheumatism (EULAR) task force was convened to develop a standardised core set for data collection in RA that should (1) harmonise data collection in future observational studies, (2) act as a common data model against which existing databases and EMR systems can be mapped and (3) ensure that new data collections in routine clinical practice can support the generation of future research-quality data.

\section{METHODS}

The task force comprised a steering committee $(\mathrm{n}=10$, including two co-conveners (WD and JA), a EULAR methodologist (LG), a patient partner (CZ) and three fellows (HR, KC and $\mathrm{EN})$ ), a working group (additional 15 experts from 11 European countries and the USA, including patients, clinical researchers and experts in the area of epidemiology, quality of care and common data modelling, physicians and other health professionals) and a pan-European expert panel $(\mathrm{n}=90)$.

The task force employed a stepwise process (figure 1) in accordance with the EULAR standard operating procedure ${ }^{4}$ underpinned by an inventory of data collection in existing RA clinical cohorts and registers. ${ }^{1}$

a. An hierarchical literature review was carried out by the fellows in PubMed to identify data items and instruments used in existing RA registers and clinical cohorts and the frequency with which these items and instruments were collected. Results were cross-checked with EULAR recommendations for reporting in RA clinical trials and trial extension studies ${ }^{2}$ 


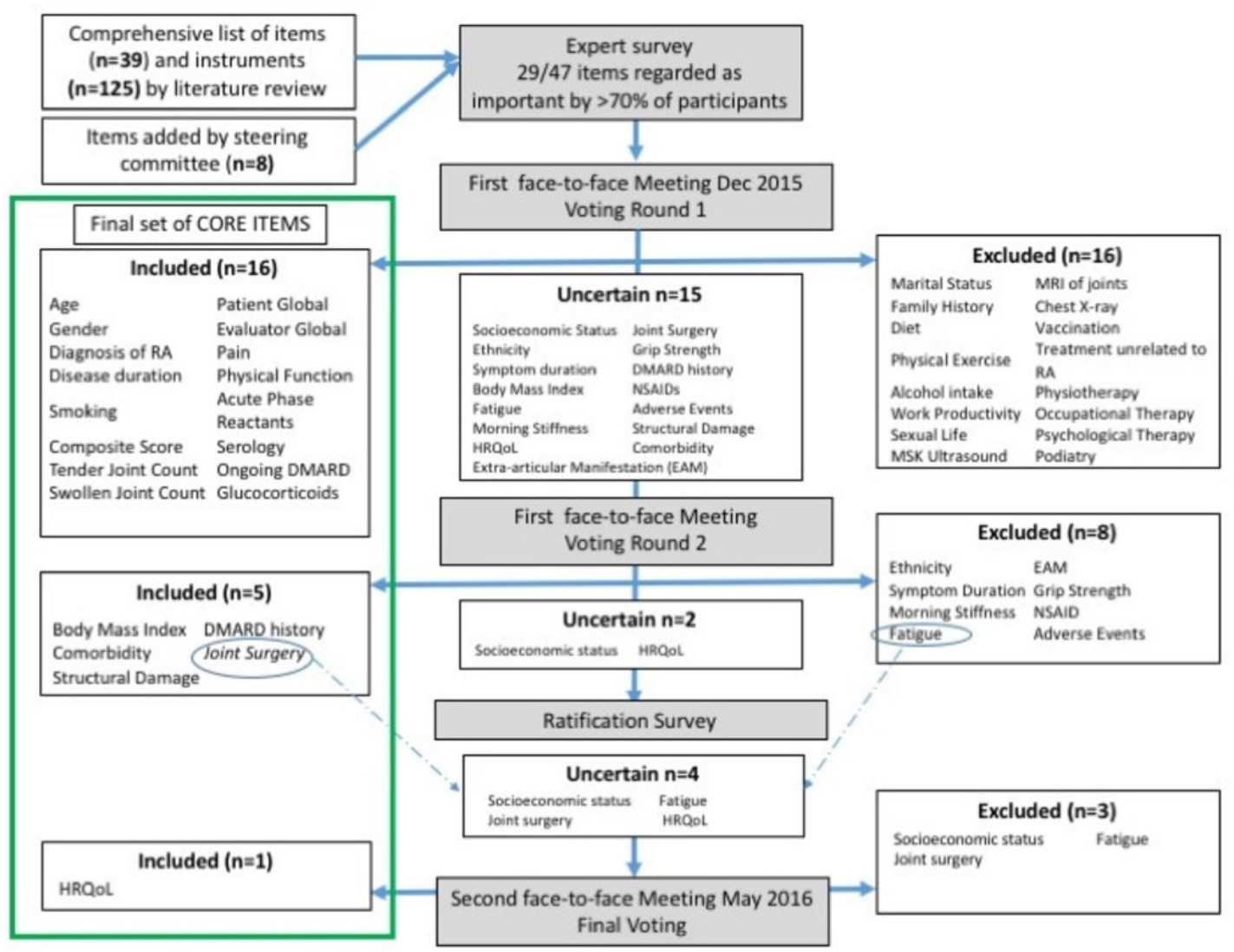

Figure 1 Flow chart of the multistep process of the project. DMARD, disease-modifying antirheumatic drugs; HRQol, health-related quality of life; MSK, musculoskeletal; NSAID, non-steroidal anti-inflammatory drug; RA, rheumatoid arthritis.

and with the EULAR outcome measures library. Items and instruments felt to be missing could be added by the steering committee.

b. In an online survey, the expert panel rated the perceived importance of each of the above items (and instruments) to be included in a core set, using a grading scale from 1 (not important) to 9 (very important). Again, items and instruments could be added.

c. At the first face-to-face meeting of the working group, electronic voting on individual items and instruments to include in the core set took place. We defined consensus as $\geq 70 \%$ of participants voting either YES or NO to the inclusion of a given item; if no consensus was reached, a second vote took place after a brief discussion which was led by an independent moderator (DP). In this second round, the threshold for consensus was lowered to $60 \%$. The resultant list of items that had reached consensus for inclusion informed a single round of voting on the instruments with which to collect information on the items.

d. Additional information on items and instruments for which no consensus was reached was collected. Ratification of items included, reasons for any exclusion and voting on items that had not reached consensus at the face-to-face meeting was performed via an online survey within the working group.

e. At the second face-to-face meeting of the working group, voting was conducted for outstanding items, then for final approval of the complete core list of items, followed by voting on instruments for all items in the final list using the same method of moderated discussion and voting cut-offs as outlined above.
In all voting processes, the panellists were reminded of the importance of striking a balance between clinical feasibility and the potential to generate research-quality data.

\section{RESULTS}

\section{Literature review}

Published articles from 67 different European RA registers and clinical cohorts were included. In total, 39 items and 125 instruments were identified; eight additional items for consideration were added (see tables 1 and 2 in the online supplementary file 1).

\section{Online survey}

Ninety experts from 28 different European countries including patients (18\%), allied health professionals (18\%), physicians $(55 \%)$ and researchers $(10 \%)$ participated in the survey. Twenty-nine of the 47 proposed items were considered important enough for inclusion into a core set (see table 2 in the online supplementary file 1 ).

\section{First face-to-face meeting}

Twenty-one out of 25 (84\%) members attended. In the first voting round, consensus for inclusion was reached for 16/47 (34\%) items and consensus for exclusion for 16/47 (34\%) items. After discussion and a second round of voting on the remaining 15 items, consensus was reached for inclusion of 5 and for exclusion of 7, leaving two items ('health-related quality of life' and 'socioeconomic status') without consensus (see table 2 in the online supplementary file 1 ). 


\section{Online ratification}

Twenty-three out of 25 (92\%) members participated. The voting results from the face-to-face meeting were confirmed except for socioeconomic status, health-related quality of life, fatigue and joint surgery (lack of consensus).

\section{Second face-to-face meeting}

Seventeen out of 25 (68\%) members attended. Voting led to the exclusion of items 'fatigue', 'joint surgery' and 'socioeconomic status' but inclusion of 'health-related quality of life'. Next, the resultant set of the remaining 21 items was voted on in its entirety and $100 \%$ of the participants agreed (see table 1 in the online supplementary file 1). Moderated discussion and sequential voting on the instruments with which to collect these 21 items resulted in consensus for all but two instruments (how to collect data for items 'glucocorticoids' and 'comorbidity').

\section{Second online survey}

A final ad hoc online survey with prespecified suggestions provided by the steering committee for the outstanding instruments for items 'glucocorticoids' and 'comorbidity' was performed (see table 3 in the online supplementary file 1 ). Twenty-one out of 25 (84\%) members participated and consensus was reached. The final core set thus contained 21 items, each with its preferred instrument (see table 1).

\section{DISCUSSION}

This EULAR task force has defined an RA core set, including both items and instruments to support standardised RA data collection in clinical practice and research. This will enable collaborative research studies and increase comparability across studies.

Unlike most previous core sets, this set was specifically developed keeping clinical feasibility in mind. Importantly, 'core' underscores that the set represents a minimum, acknowledging that individual stakeholders are likely to add items or instruments of particular interest to their own data collection.

Consensus for inclusion was straightforward for the majority of items: 16 of the final 21 items were agreed in the first round of voting. Six additional items were included, and after a ratification survey and discussion, 'joint surgery' was excluded in the subsequent process. Discussion for each item is summarised in table 3 in the online supplementary file 1 . Of the 26 items ultimately excluded from the core data set, nine were seen as important by the expert group. One item, 'fatigue', was excluded from our core data set, yet is present in the Outcome Measures in Rheumatology (OMERACT) core outcome set for RA trials. ${ }^{6}$ Discussion during the meeting acknowledged that this item was important but depending on many factors unrelated to RA and can be captured by the patient global assessment of disease activity (PGA). The group repeatedly revisited the scope of a core set and agreed that fatigue was a good example of an item that might regularly be collected in addition to the core set for either clinical practice or research.

The task force was established to include expertise in registers and observational research, clinical practice, use of EMRs, the patient perspective, as well as methodological expertise in the development of core data sets ${ }^{7}$ to enable robustness and transparency. To ensure inclusiveness, all national European rheumatology societies were invited to contribute. Dissemination of the core set will be facilitated by publication online (EULAR website) and via national societies.
Table 1 Structure and content of the RA core set developed by the EULAR Task Force

\begin{tabular}{|c|c|c|}
\hline S. No & Item & $\begin{array}{l}\text { Recommendation of standardised way of } \\
\text { assessment }\end{array}$ \\
\hline 1 & Age & Date of birth \\
\hline 2 & Gender & Male/female \\
\hline 3 & Disease duration & Date of diagnosis \\
\hline 4 & Diagnosis of RA & Rheumatologist reported diagnosis of RA \\
\hline 5 & Body mass index & Weight and height \\
\hline 6 & Smoking & Current/previous/never \\
\hline 7 & Tender joints & 28 joint count \\
\hline 8 & Swollen joints & 28 joint count \\
\hline 9 & Patient global & $\begin{array}{l}\text { Measured on VAS or NRS scale capturing: (1) } \\
\text { global assessment of disease activity; (2) related to } \\
\text { arthritis and (3) today } \\
\text { Example wording: 'Considering all the ways your } \\
\text { arthritis has affected you, how do you feel your } \\
\text { arthritis is today?' anchors: excellent-very poor }\end{array}$ \\
\hline 10 & Evaluator global & $\begin{array}{l}\text { Measured on VAS or NRS scale capturing: (1) } \\
\text { global assessment of disease activity; (2) related to } \\
\text { arthritis and (3) today } \\
\text { Example wording: 'What is your overall assessment } \\
\text { of the patient's RA disease activity today?' anchors: } \\
\text { excellent-very poor }\end{array}$ \\
\hline 11 & Pain & $\begin{array}{l}\text { Measured on VAS or NRS capturing: (1) pain; ( } 2 \text { ) } \\
\text { related to arthritis and (3) last week } \\
\text { Example wording: 'How much pain did you have due } \\
\text { to your arthritis last week?' anchors: no pain-worst } \\
\text { imaginable pain }\end{array}$ \\
\hline 12 & Physical function & Health Assessment Questionnaire \\
\hline 13 & $\begin{array}{l}\text { Health-related } \\
\text { quality of life }\end{array}$ & Euro-Qol 5 dimensions* \\
\hline 14 & Composite scores & $\begin{array}{l}\text { Collection of core items } 7-12 \text { and } 15 \text { enables to } \\
\text { calculate following composite scores: } \\
\text { Clinical Disease Activity Index } \\
\text { Simplified Disease Activity Index } \\
\text { Disease Activity Score } 28 \text { joints } \\
\text { EULAR response criteria } \\
\text { American College of Rheumatology response } \\
\text { criteria }\end{array}$ \\
\hline 15 & $\begin{array}{l}\text { Acute phase } \\
\text { reactants }\end{array}$ & $\begin{array}{l}\text { C-reactive protein AND erythrocyte sedimentation } \\
\text { rate }\end{array}$ \\
\hline 16 & Serology & $\begin{array}{l}\text { Rheumatoid factor AND anticyclic citrullinated } \\
\text { peptide antibodies }\end{array}$ \\
\hline 17 & Structural damage & Presence of erosions on X-ray, Yes/No \\
\hline 18 & DMARD history & $\begin{array}{l}\text { Name of previous DMARD(s) (including biological, } \\
\text { synthetic and targeted synthetic DMARDs) }\end{array}$ \\
\hline \multirow[t]{3}{*}{19} & \multirow{3}{*}{$\begin{array}{l}\text { Ongoing/most recent } \\
\text { DMARD }\end{array}$} & Name of DMARD \\
\hline & & Start and stop date \\
\hline & & Reason for stopping \\
\hline 20 & Glucocorticoids & $\begin{array}{l}\text { Current use of oral glucocorticoids: (1) dose taken } \\
\text { today__mg prednisolone equivalent and ( } 2 \text { ) } \\
\text { continuous ( }>3 \text { months) intake YES/NO }\end{array}$ \\
\hline 21 & Comorbidities & $\begin{array}{l}\text { Binary assessment YES/NO of the six EULAR } \\
\text { comorbidity domains (cardiovascular disease, } \\
\text { malignancies, infections, gastrointestinal disease, } \\
\text { osteoporosis, depression) }{ }^{5}\end{array}$ \\
\hline
\end{tabular}

${ }^{*}$ Free licence available after registration for non-commercial parties according to www.euroqol.org.

DMARD, disease-modifying antirheumatic drugs; EULAR, European League Against Rheumatism; NRS, numerical rating scale; RA, rheumatoid arthritis; VAS, visual analogue scale.

Following agreement on this core set, it is important that it is adopted by future observational data collections and research studies in RA. For EMR systems to deliver to RA research, the 
core set also needs to be integrated into EMR systems. Additional clinical benefits of collection of structured data such as informing consultations, viewing longitudinal disease progression, supporting audit and benchmarking care quality ${ }^{8}$ should be recognised and may encourage its clinical adoption.

In summary, through a multistep, multistakeholder and evidence-based process, this task force has developed an RA core set that may (1) harmonise data collection in future observational studies, (2) act as a common data model against which existing databases can be mapped and (3) serve as a template for standardised data collection in routine clinical practice to support the generation of research-quality data. This effort, which will continue to be updated and revised after evaluation of its implementation, may serve as an example for other conditions beyond RA.

\section{Author affiliations}

'Department of Internal Medicine III, Division of Rheumatology, Medical University Vienna, Vienna, Austria

2Department of Rheumatology, Karolinska University Hospital, Stockholm, Sweden

${ }^{3}$ Department of Medicine Solna, Karolinska Institutet, Stockholm, Sweden

${ }^{4}$ Academic Rheumatology Department, King's College London, London, UK

${ }^{5}$ Rheumatology Department, Sorbonne Universités, UPMC University Paris, Paris, France

${ }^{6}$ Department of Rheumatology, AP-HP, Hopital Pitie-Salpetriere, Paris, France ${ }^{7}$ NIHR Manchester Musculoskeletal Biomedical Research Unit, Central Manchester NHS Foundation Trust, Manchester Academic Health Science Centre, Manchester, UK ${ }^{8}$ Patient Partner of Romanian League Against Rheumatism, Bucharest, Romania ${ }^{9}$ Department of Patient and Care, Maastricht University Medical Centre, Maastricht, The Netherlands

${ }^{10}$ Department of Biostatistics, MRC North West Hub for Trials Methodology Research, University of Liverpool, Liverpool, UK

${ }^{11}$ Research Center of Rheumatic Diseases, Research Center of Rheumatic Diseases,

'Sf. Maria' Hospital, University of Medicine and Pharmacy 'Carol Davila', Bucharest, Romania

${ }^{12}$ Department of Rheumatology and Clinical Immunology, Charité-University Medicine Berlin, Berlin, Germany

${ }^{13}$ Instituto de Salud Musculoesquelética, Instituto de Salud Musculoesquelética, Madrid, Spain

${ }^{14}$ Medicine Faculty, APHP, Rheumatology B Department, Paris Descartes University, Cochin Hospital, Paris, France

${ }^{15}$ Division of Rheumatology, Department of Internal Medicine Specialties, University Hospitals of Geneva, Geneva, Switzerland

${ }^{16}$ Department of Rheumatology, Martina Hansens Hospital, Gjettum, Norway

${ }^{17}$ Norwegian University of Science and Technology, Trondheim, Norway

${ }^{18}$ Center for Rheumatology and Spine Diseases, Centre of Head and Orthopaedics,

Copenhagen Center for Arthritis Research, Rigshospitalet, Glostrup, Denmark

${ }^{19}$ Department of Clinical Medicine, Faculty of Health and Medical Sciences, University

of Copenhagen, Copenhagen, Denmark

${ }^{20}$ Department of Rheumatology, North Devon, UK

${ }^{21}$ Rheumatology Department, Gartnavel General Hospital, Glasgow, UK

${ }^{22}$ Institute of Inflammation and Ageing, Institute of Inflammation and Ageing,

Arthritis Research UK Rheumatoid Arthritis Pathogenesis Centre of Excellence and

MRC Arthritis Research UK Centre for Musculoskeletal Ageing Research, University of

Birmingham, Birmingham, UK

${ }^{23}$ Biomedical Research Centre, NIHR Birmingham, Birmingham, UK

${ }^{24}$ Sandwell and West Birmingham Hospitals NHS Trust, Birmingham, UK

${ }^{25}$ Department of Biomedical Informatics, Columbia University, New York, USA
${ }^{26}$ Janssen Research and Development, Janssen, Titusville, USA

${ }^{27}$ Department of Rheumatology, Hospital Garcia de Orta and Instituto de Medicina

Molecular da Faculdade de Medicina, Universidade de Lisboa, Lisboa, Portugal

${ }^{28}$ Department of Rheumatology, Leiden University Medical Center, Leiden, The

Netherlands

${ }^{29}$ Department of Rheumatology, Erasmus Medical Center, Rotterdam, The

Netherlands

${ }^{30}$ Department of Rheumatology, Bernhoven, Uden, The Netherlands

${ }^{31}$ Patient Research Partner, Paris, France

${ }^{32}$ Institute of Rheumatology, Institute of Rheumatology, Prague, Czech Republic

${ }^{33}$ Department of Rheumatology, First Faculty of Medicine, Charles University, Prague, Czech Republic

${ }^{34}$ Arthritis Research UK Centre for Epidemiology, Manchester Academic Health

Sciences Centre, The University of Manchester, Manchester, UK

Acknowledgements The authors want to thank all contributing experts who participated in the online survey.

Contributors All authors listed fulfill authorship criteria. They have contributed to the task force being part of the working group. They have been engaged in the conception of the work, attended meetings and were involved in drafting the manuscript.

Funding This project was funded by a EULAR project grant.

Competing interests None declared.

Provenance and peer review Not commissioned; externally peer reviewed.

(C) Article author(s) (or their employer(s) unless otherwise stated in the text of the article) 2018. All rights reserved. No commercial use is permitted unless otherwise expressly granted.

\section{REFERENCES}

1 Radner H, Dixon W, Hyrich K, et al. Consistency and utility of data items across european rheumatoid arthritis clinical cohorts and registers. Arthritis Care Res 2015;67:1219-29.

2 Aletaha D, Landewe R, Karonitsch T, et al. Reporting disease activity in clinical trials of patients with rheumatoid arthritis: EULAR/ACR collaborative recommendations. Ann Rheum Dis 2008;67:1360-4.

3 Buch MH, Silva-Fernandez L, Carmona L, et al. Development of EULAR recommendations for the reporting of clinical trial extension studies in rheumatology. Ann Rheum Dis 2015;74:963-9.

4 van der Heijde D, Aletaha D, Carmona L, et al. 2014 Update of the EULAR standardised operating procedures for EULAR-endorsed recommendations. Ann Rheum Dis 2015:74:8-13.

5 Baillet A, Gossec L, Carmona L, et al. Points to consider for reporting, screening for and preventing selected comorbidities in chronic inflammatory rheumatic diseases in daily practice: a EULAR initiative. Ann Rheum Dis 2016;75:965-73.

6 Kirwan JR, Minnock P, Adebajo A, et al. Patient perspective: fatigue as a recommended patient centered outcome measure in rheumatoid arthritis. J Rheumatol 2007:34:1174-7.

7 Kirkham JJ, Gorst S, Altman DG, et al. Core outcome Set-STAndards for reporting: the COS-STAR statement. PLoS Med 2016;13:e1002148.

8 Ibfelt EH, Jensen DV, Hetland ML. The Danish nationwide clinical register for patients with rheumatoid arthritis: DANBIO. Clin Epidemiol 2016;8:737-42.

9 Nikiphorou E, Radner H, Chatzidionysiou K, et al. Patient global assessment in measuring disease activity in rheumatoid arthritis: a review of the literature. Arthritis Res Ther 2016;18:251.

10 Felson DT, Smolen JS, Wells G, et al. American College of Rheumatology/European League against Rheumatism provisional definition of remission in rheumatoid arthritis for clinical trials. Ann Rheum Dis 2011;70:404-13.

11 van der Heijde D, van der Helm-van Mil AH, Aletaha D, et al. EULAR definition of erosive disease in light of the 2010 ACR/EULAR rheumatoid arthritis classification criteria. Ann Rheum Dis 2013;72:479-81. 